\title{
Assessment of Human Skeletal Muscle Contraction and Force by Diffusion Tensor Imaging
}

\author{
Junichi Hata ${ }^{1,2,3 *}$, Haruyuki Nagata3 ${ }^{3}$, Kazuki Endo ${ }^{3}$, Yuji Komaki1,2, Masakazu Sato3, \\ Tomokazu Numano ${ }^{3}$, Kazuo Yagi ${ }^{3}$ \\ ${ }^{1}$ Department of Physiology, School of Medicine, Keio University, Tokyo, Japan \\ ${ }^{2}$ Central Institute for Experimental Animals, Kawasaki, Japan \\ ${ }^{3}$ Graduate School of Human Health Science, Tokyo Metropolitan University, Tokyo, Japan \\ Email: "j.hata@a3.keio.jp
}

Received 28 October 2015; accepted 1 December 2015; published 4 December 2015

Copyright (C) 2015 by authors and Scientific Research Publishing Inc.

This work is licensed under the Creative Commons Attribution International License (CC BY). http://creativecommons.org/licenses/by/4.0/

(c) (i) Open Access

\section{Abstract}

We aimed to investigate the association between mobility and skeletal muscle strength by using magnetic resonance diffusion tensor imaging (DTI). This study included 20 healthy male volunteers (mean age, $21.8 \pm 1.1$ years). The maximum voluntary strength (MVC) of each participant was measured with the ankle joint in plantar and dorsal flexion using an instrument for measuring muscle strength. Moreover, magnetic resonance imaging (MRI) was performed with the ankle joint at rest, in plantar flexion, and in dorsal flexion. For imaging, a 1.5-T MRI device was used, and a diffusion-weighted stimulated echo-planar imaging pulse sequence. Tensor eigenvalues $(\lambda)$, fractional anisotropy $(F A)$, and the apparent diffusion coefficient $(A D C)$ were calculated from data obtained by DTI. The resulting MRI data were compared to the data on muscle mobility or strength and statistically analyzed. Regarding changes in DTI indices during muscle movements, anisotropy of the tibialis anterior was significantly increased from rest to plantar flexion $(P<0.01)$, whereas no significant change was observed in dorsal flexion (n.s.). In contrast, the extent of significant changes in anisotropy of the medial gastrocnemius (mGC) and soleus (SOL) was small at plantar flexion (mGC, $P<0.01$; SOL, n.s.), whereas the indices were significantly increased at dorsal flexion $(P<0.01)$. Regarding the correlation between MVC of each skeletal muscle and the DTI indices, $F A$ and $\lambda_{3}$ were significantly correlated in movements involving the muscles, whereas no significant correlation was observed in movements not involving them. Changes in intramuscular water molecules by elongation and contraction of the skeletal muscle fibers could be assumed to affect changes in diffusional anisotropy. When muscles contract, the space between myocytes was reduced and they might become increasingly dense. Moreover, diffusional anisotropy increased with

\footnotetext{
*Corresponding author.
}

How to cite this paper: Hata, J., Nagata, H., Endo, K., Komaki, Y., Sato, M., Numano, T. and Yagi, K. (2015) Assessment of Human Skeletal Muscle Contraction and Force by Diffusion Tensor Imaging. Open Journal of Radiology, 5, 189-198. 
increasing MVC, whereas $A D C$ remained unchanged. DTI was suggested to produce measurements similar to the degree of muscle strength.

\title{
Keywords
}

\author{
Diffusion, Tensor, Skeletal Muscle, Contraction, Muscle Force
}

\section{Introduction}

Diffusion tensor imaging (DTI) is a technique that allows calculation of the diffusion direction of water molecules into the body [1]. Fractional anisotropy (FA) can be calculated from values called apparent diffusion coefficient $(A D C)$ determined by measuring the long axis of tissue $\left(\lambda_{1}\right)$ and two vertical lines to the axis $\left(\lambda_{2}\right.$ and $\left.\lambda_{3}\right)$. This FA is considered extremely useful for assessing nerve-like fibrous tissue [2]-[4]. In recent years, with advances in the magnetic field gradient system and the application of a high magnetic field, FA has become applicable to many body tissues including not only central nervous areas with relatively long T2 values (90 ms) but also tissues with short $\mathrm{T} 2$ values (e.g. hepatic parenchyma [ $40 \mathrm{~ms}$ ], skeletal muscles [50 ms], and cardiac muscle [60 ms]). In particular, the skeletal muscle structure, which appears as an extremely long and narrow fiber, seems to be a DTI-compatible body tissue.

The skeletal muscles are directly associated with quality of life (QOL) and activities of daily living (ADL) and comprise a medically important organ that is affected by age-related disease and mobility impairments. Assessments of the skeletal muscles are considered important in the field of sports medicine. Various methods used to assess the skeletal muscles include electromyography, muscle biopsy, ultrasonography, computed tomography (CT), and magnetic resonance imaging (MRI). In particular, MRI is attracting increasing attention as a modality that allows noninvasive three-dimensional assessment. Many studies of the skeletal muscles using MRI have been reported, including assessments of muscle morphology [5] [6], muscle metabolism with hydrogen $(1 \mathrm{H})$ or phosphorus (31P) by magnetic resonance spectroscopy (MRS) [7]-[9], and muscle function by the measurement of T2 values [10]-[13]. DTI, on which the present study focuses, has also been reported in studies on the assessment of muscle morphology, such as measurement of pennation angle by tensor tractography [14], depiction of orientation [15]-[17], and assessment of muscle damage [18].

Studies using DTI on skeletal muscles include many reports on the assessment of muscle morphology, whereas few have examined muscle function. The motor function of the skeletal muscles is associated with contraction and relaxation of muscle fibers, in other words, changes in muscle fiber structure. The ability to measure muscle morphology with DTI suggests that muscle function can also be assessed by a detailed assessment of the measurements. In 2005, Heemskerk et al., who studied mice, reported that the physiological cross-sectional area (PCSA) and $\lambda_{3}$ of the skeletal muscles were in a proportional relationship [14]. In addition, it has long been reported that muscle strength also greatly affects the PCSA of the skeletal muscles involved in movement [19]. However, there has been no report of data associating muscle strength with FA and eigenvalues, which can be calculated using DTI.

In the present study of healthy volunteers, we measure fluctuations in DTI indices during skeletal muscle contraction and relaxation using DTI and also aim to reveal the correlation between the indices and muscle strength.

\section{Material and Methods}

The present study was approved by the ethics committee of our institution and included 20 healthy adult male volunteers who received a sufficient explanation of the experiment and provided informed consent. The subjects for the experiment were chosen using a bulletin board inside the college. Their characteristics were as follows: age, $21.8 \pm 1.1$ years (mean \pm standard deviation); height, $1.72 \pm 0.05 \mathrm{~m}$; weight, $59.4 \pm 7.8 \mathrm{~kg}$; and body mass index (BMI), $20.0 \pm 5.1 \mathrm{~kg} / \mathrm{m}^{2}$. The imaging sites were the skeletal muscles of the lower leg (tibialis anterior [TA], soleus [SOL], and medial gastrocnemius [mGC]). The inclusion criteria were no current or previous treatment for any disorder of the sites and no regular exercise. The study period took three months to scan MRI and two months for analysis. 
1) Exercise tolerance

The participants lay in the supine position with the knee joints completely extended for MRI, and the exercise positions included the ankle joint at rest, dorsal flexion, and plantar flexion. For MRI of the ankle joint at dorsal and plantar flexion, the ankle joint was held at 50\% maximum voluntary contraction (MVC). Moreover, MVC was measured prior to imaging. During exercise, the distal portion of the knee joint and the proximal portion of the ankle joint were fixed to prevent the imaging sites from departing from the predetermined imaging range. MRI and measurement of the MVC were performed with the participants in the same posture by the same fixation. For the measurement, we used a custom made myodynamometer for the lower leg that we developed from a Molten-made digital myodynamometer for the lower limb.

2) MR imaging

For MRI, a 1.5-Tesla magnetic resonance scanner (Signa Horizon Lx Ver. 9.0; General Electric Healthcare, Tokyo, Japan) and knee coil (General Electric Healthcare) were used. On this device, the maximum magnetic field gradient amplitude is $22 \mathrm{mT} / \mathrm{m}$, while the maximum slew rate is $77 \mathrm{mT} / \mathrm{m} / \mathrm{m}$. The imaging range of the lower leg was an area around the greatest diameter of the lower leg.

For the DTI pulse sequence, we used the single-shot diffusion-weighted stimulated-echo echo-planar imaging (DW-STE EPI) pulse sequence that we had independently developed (Figure 1). The DW-STE EPI method enables investigators to obtain high signals even in tissues with extremely short T2 values (e.g. hepatic parenchyma [40 ms], skeletal muscles [50 ms], and cardiac muscle [60 ms]) [20] [21].

The imaging parameters used in the present study were as follows: repetition time (TR)/echo time (TE)/mixing time (TM), 4000/44.1/208.2 ms; $\Delta / \delta, 225.9 / 11.4 ; b$-value, $800 \mathrm{~s} / \mathrm{mm}^{2}$; number of excitations (NEX), 8; field of view (FOV), $240 \times 240 \mathrm{~mm}^{2}$; matrix size, $128 \times 128$; number of slices, 6; slice thickness, 8 mm with no gap; motion probing gradient (MPG) moment, six axes (xy, xz, yz, $-\mathrm{xy},-\mathrm{xz},-\mathrm{yz}$ ); and total DW-STE scanning time, $224 \mathrm{~s}$. To determine the anatomical locations of the skeletal muscles, T1-spoiled gradient recalled acquisition in the steady state (SPGR) was used. The parameters were as follows: TR/TE, 20/5 ms; frip angle, $40^{\circ}$; NEX, 1 ; FOV, $240 \times 240 \mathrm{~mm}^{2}$; matrix size, $128 \times 128$; number of slices, 5; and slice thickness, $8 \mathrm{~mm}$ with no gap. The slice center was set at the greatest diameter of the lower leg on the sagittal localized images.

3) Diffusion tensor processing

Imaging data were processed by a free software provided by the University of Tokyo (dTV2, currently unavailable). Tensor analysis of the signals of each vector component yielded three eigenvalues corresponding to the axes of the tensor ellipsoid $\left(\lambda_{1}, \lambda_{2}\right.$, and $\left.\lambda_{3}\right)$. From these eigenvalues, the apparent diffusion coefficient $(A D C)$ was calculated using the following equation [22]:

$$
A D C=\frac{\lambda_{1}+\lambda_{2}+\lambda_{3}}{3}
$$

The fractional anisotropy (FA) was calculated using the following equation [22]:

$$
F A=\sqrt{\frac{3}{2}} \cdot \frac{\sqrt{\left(\lambda_{1}-\bar{\lambda}\right)^{2}+\left(\lambda_{2}-\bar{\lambda}\right)^{2}+\left(\lambda_{3}-\bar{\lambda}\right)^{2}}}{\sqrt{\lambda_{1}^{2}+\lambda_{2}^{2}+\lambda_{3}^{2}}}
$$

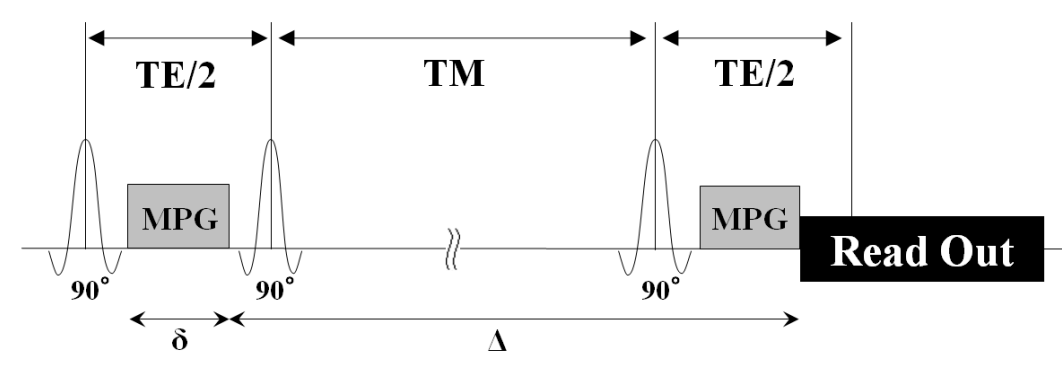

Figure 1. Single shot DW-STE EPI pulse sequence diagram. This pulse sequence is based on the stimulated-echo method. It employs three 90-degree radio frequency (RF) pulses. Magnetization is once stored longitudinally by the second RF pulse, and echo is converged by the third RF pulse. In addition, diffusion contrast is generated by applying motion probing gradient (MPG) between the first and second RF pulses and between the third RF pulse and readout gradient. 
While TA, mGC, and SOL in the imaged lower leg were targeted for measurement, the regions of interest (ROI) were carefully selected on T1-weighted SPGR images (Figure 2). Each ROI was applied to the calculated DTI images $\left(F A, \lambda_{1}, \lambda_{2}, \lambda_{3}\right.$, and $A D C$ ) to measure the indices. Image $\mathrm{J}$ software version 1.37 (National Institutes of Health) was used for the ROI selection and measurement.

4) Statistical processing

To test for significant differences in changes in the DTI indices due to muscle movement, the nonparametric Wilcoxon signed-rank test was performed with a significance level of 5\%. Moreover, to test for correlations between MVC and DTI indices of the lower leg, the nonparametric Spearman rank correlation coefficient test was performed with a significance level of $5 \%$.

\section{Results}

Changes in the DTI indices of the skeletal muscles of the lower leg with the ankle joint positioned at dorsal flexion and rest.

The DTI indices were measured when the ankle joint was positioned at dorsal flexion and rest (Figure 3 and Table 1). The measuring sites in the ROI were the TA, mGC, and SOL. The TA was contracted, whereas the mGC and SOL were relaxed.

Table 1. Significant difference test of DTI indices at rest and during muscle movements (plantar and dorsal flexion). Each DTI index of the skeletal muscles of the lower leg derived from MRI images taken with the ankle joint at dorsal and plantar flexion was tested for significant differences upon comparison with the indices at rest.

\begin{tabular}{|c|c|c|c|c|c|c|}
\hline & & $F A$ & $\lambda_{1}$ & $\lambda_{2}$ & $\lambda_{3}$ & $A D C$ \\
\hline \multirow{2}{*}{ TA } & Dorsal flexion & 0.027 & 0.0001 & 0.0001 & 0.0008 & 0.89 \\
\hline & Plantar flexion & 0.62 & 0.82 & 0.89 & 0.91 & 0.0001 \\
\hline \multirow{2}{*}{ mGM } & Dorsal flexion & 0.004 & 0.64 & 0.004 & 0.006 & 0.0001 \\
\hline & Plantar flexion & 0.0001 & 0.0004 & 0.00008 & 0.00008 & 0.007 \\
\hline \multirow{2}{*}{ SOL } & Dorsal flexion & 0.052 & 0.026 & 0.73 & 0.76 & 0.0001 \\
\hline & Plantar flexion & 0.001 & 0.0002 & 0.0001 & 0.0001 & 0.21 \\
\hline
\end{tabular}

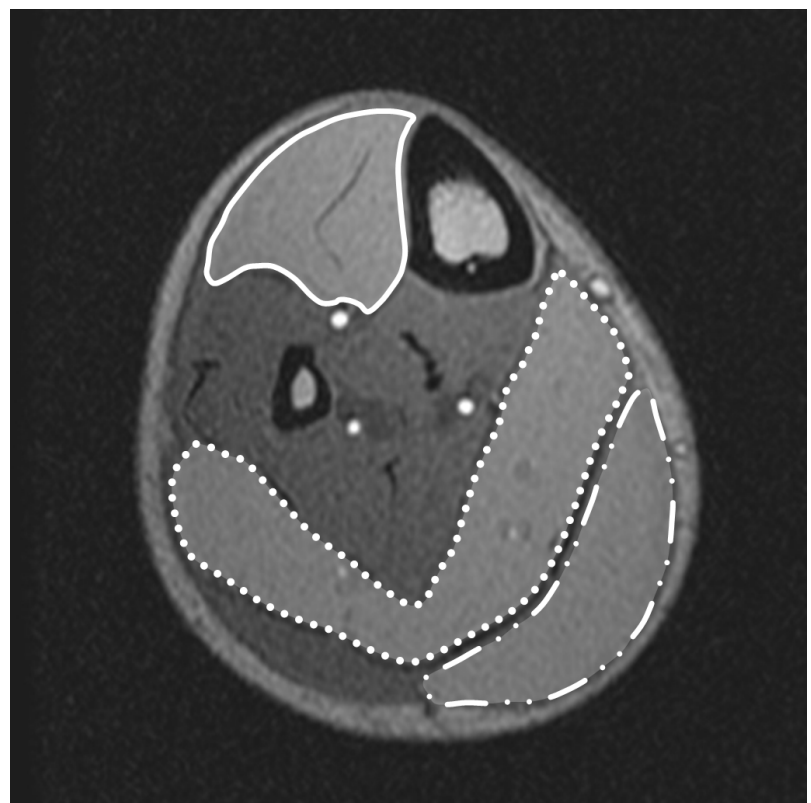

Figure 2. Setting of ROI of the skeletal muscles of the lower leg by using morphological imaging ROI was set on morphological images obtained by T1-weighted SPGR pulse sequence. The area surrounded by a solid line represents TA. The area surrounded by a dotted line represents SOL. The area surrounded by a dotted-solid line represents mGC. 

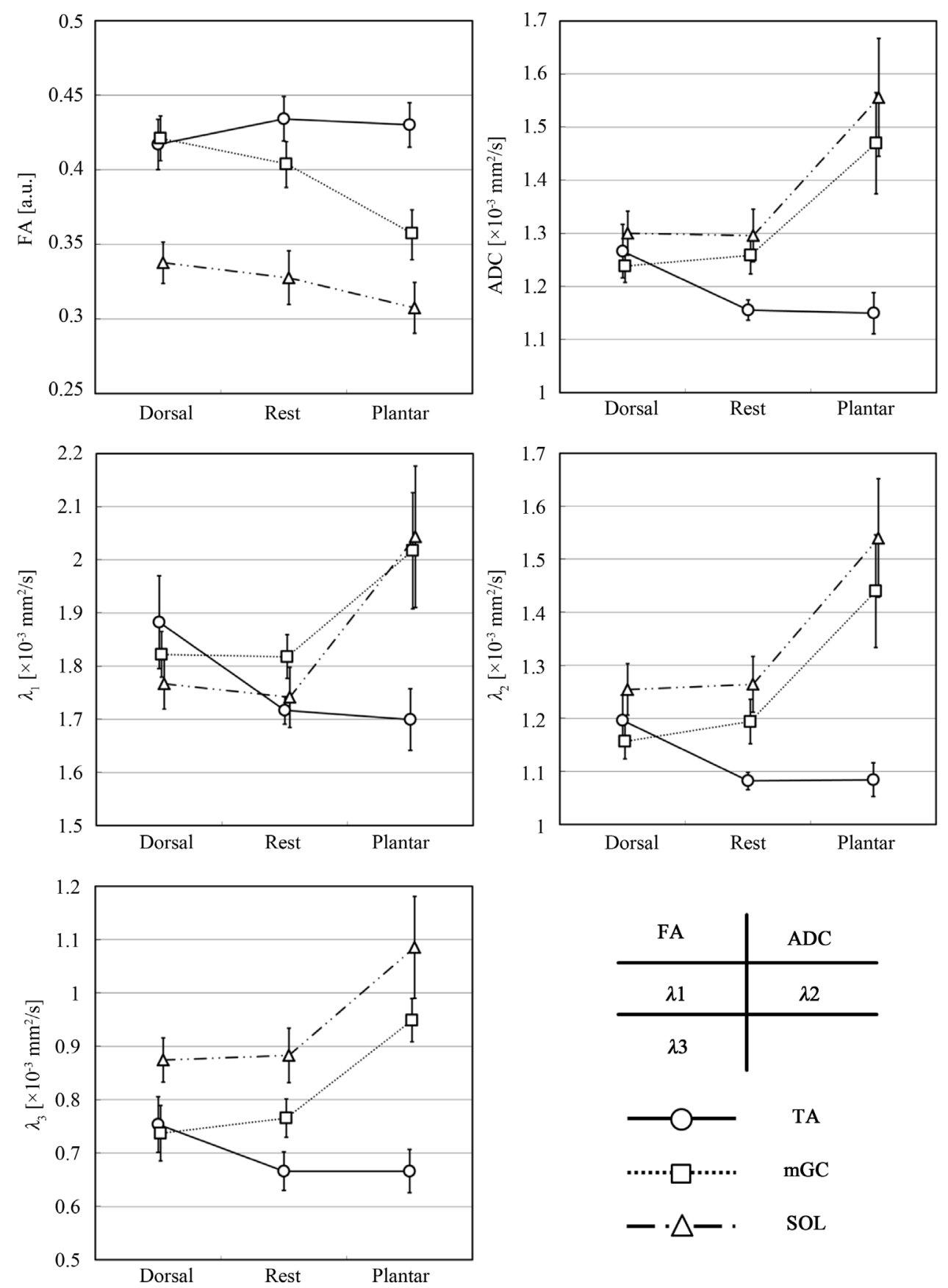

Figure 3. Fluctuations in DTI indices according to movements of the ankle joint These figures show various DTI indices derived from MRI images taken while the ankle joint was positioned at rest, plantar flexion, and dorsal flexion (upper left: FA; upper right: $A D C$; middle left: $\lambda_{1}$; middle right: $\lambda_{2}$; lower left: $\lambda_{3}$ ). The solid lines with circles represent TA. The dotted lines with squares represent mGC. The dotted-solid lines with triangles represent SOL.

In TA, $F A(-3.95 \%, P<0.05)$ was significantly decreased, whereas $\lambda_{1}(9.66 \%, P<0.01), \lambda_{2}(10.5 \%, P<$ $0.01), \lambda_{3}(13.1 \%, P<0.01)$, and $A D C(9.57 \%, P<0.01)$ were significantly increased. All of the DTI indices were significantly changed. In the mGC, $F A(4.25 \%, P<0.01)$ was significantly increased, whereas $\lambda_{2}(-3.14 \%$, $P<0.01), \lambda_{3}(-3.70 \%, P<0.01)$, and $A D C(-1.61 \%, P<0.01)$ were significantly decreased. No significant change was observed in $\lambda_{1}\left(0.248 \%\right.$, n.s.). In the SOL, $\lambda_{1}(1.46 \%, P<0.05)$ was significantly increased, whereas 
no significant change was observed in $F A(3.052 \%$, n.s. $), \lambda_{2}(-0.75 \%$, n.s, $), \lambda_{3}(-0.93 \%$, n.s.), and $A D C(0.00 \%$, n.s.).

Changes in the DTI indices of the skeletal muscles of the lower leg with the ankle joint positioned at plantar flexion and rest.

The DTI indices were measured with the ankle joint positioned at plantar flexion and rest (Figure 3 and Table 1). The measurement sites in the ROI were the TA, mGC, and SOL. The TA was relaxed, whereas the mGC and SOL were contracted.

The TA showed no significant change in any of the DTI indices, namely $F A\left(-0.96 \%\right.$, n.s.), $\lambda_{1}(-1.00 \%$, n.s.), $\lambda_{2}\left(0.20 \%\right.$, n.s.), $\lambda_{3}(-0.03 \%$, n.s.), and $A D C(-0.50 \%$, n.s. $)$. In the mGC, FA $(-11.5 \%, P<0.01)$ was significantly decreased, whereas $\lambda_{1}$ (10.9\%, $\left.P<0.01\right)$, $\lambda_{2}$ (20.6\%, $\left.P<0.01\right), \lambda_{3}$ (23.9\%, $\left.P<0.01\right)$, and $A D C(16.7 \%, P<$ $0.01)$ were significantly increased. Significant changes were observed in all of the DTI indices. In the SOL, FA (-6.15\%, $P<0.01)$ was significantly decreased, whereas $\lambda_{1}$ (17.4\%, $\left.P<0.01\right), \lambda_{2}(21.8 \%, P<0.01), \lambda_{3}(22.9 \%$, $P<0.01)$, and $A D C(19.7 \%, P<0.01)$ were significantly increased. Significant changes were observed in all of the DTI indices.

\section{Association between Skeletal Muscle Strength of the Lower Leg and the DTI Indices}

The MVC and DTI indices of the leg were compared and assessed. Statistical analysis (Figure 4 and Table 2)
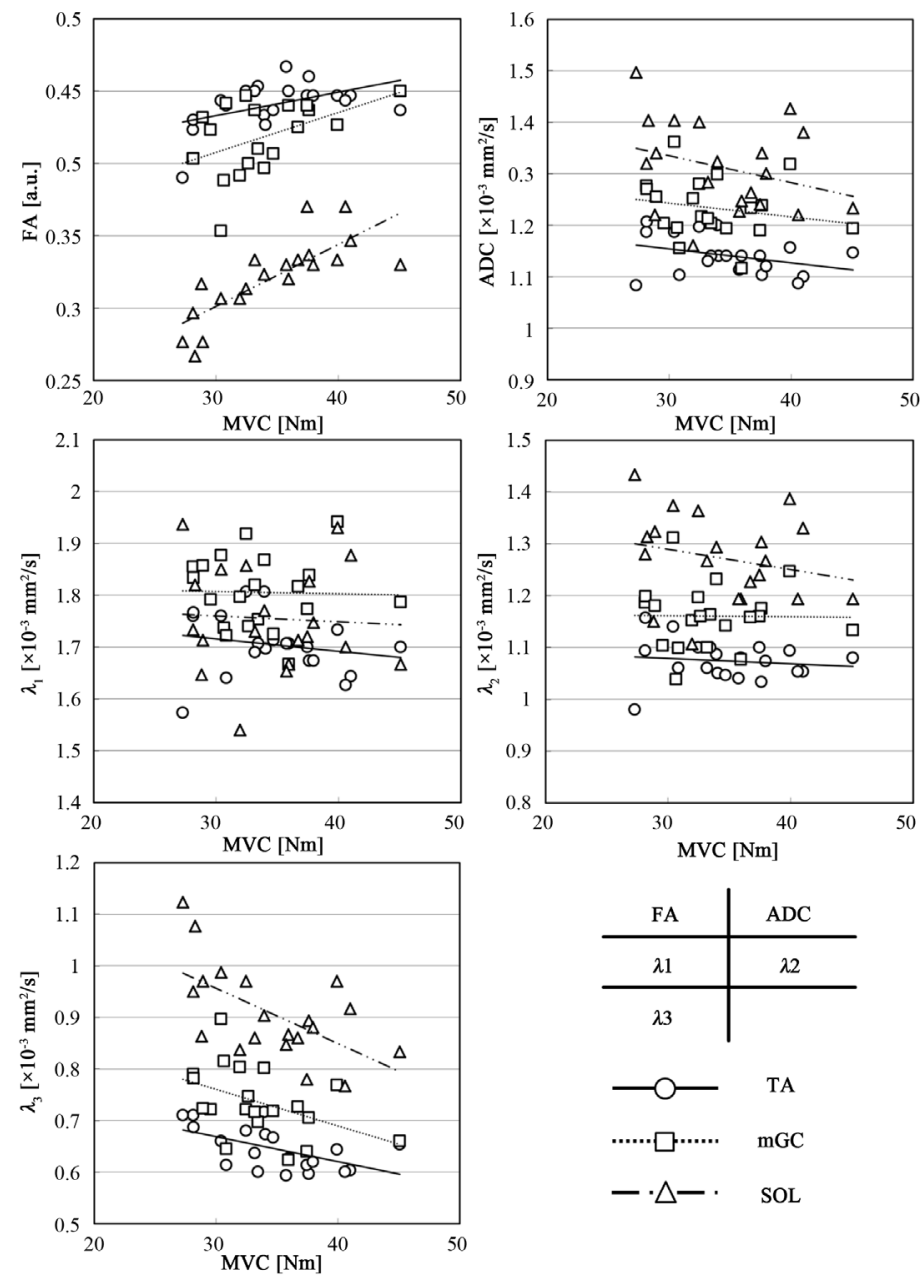

Figure 4. Association of maximum voluntary contraction with each DTI index. The vertical axes of these figures show each DTI index at rest, and the horizontal axes show MVC (upper left: $F A$; upper right: $A D C$; middle left: $\lambda_{1}$; middle right: $\lambda_{2}$; lower left: $\lambda_{3}$ ). The solid lines with circles represent TA. The dotted lines with squares represent mGC. The dotted-solid lines with triangles represent SOL. 
Table 2. Correlation analysis of strength of the skeletal muscles of the lower leg and DTI indices. Strength of the skeletal muscles of the lower leg (MVC) was analyzed for correlation with each DTI index.

\begin{tabular}{cccccccc}
\hline & $F A$ & $\lambda_{1}$ & $\lambda_{2}$ & $\lambda_{3}$ & $A D C$ \\
\hline \multirow{3}{*}{ TA } & Dorsal flexion & 0.7 & 0.11 & 0.04 & 0.67 & 0.31 \\
& Plantar flexion & 0.23 & 0.19 & 0.12 & 0.45 & 0.33 \\
\multirow{3}{*}{ mGM } & Dorsal flexion & 0.17 & 0.36 & 0.16 & 0.32 & 0.33 \\
& Plantar flexion & 0.47 & 0.02 & 0.01 & 0.44 & 0.19 \\
& Dorsal flexion & 0.62 & 0.17 & 0.22 & 0.56 & 0.32 \\
& Plantar flexion & 0.78 & 0.05 & 0.23 & 0.59 & 0.3 \\
\hline
\end{tabular}

revealed that, in the TA at dorsal flexion, MVC was significantly correlated with $F A(r=0.701, P<0.01)$ and $\lambda_{3}$ ( $r=0.675, P<0.01$ ) but not with $\lambda_{2}, \lambda_{3}$, and $A D C$. In the TA at plantar flexion, a significant difference was observed in $\lambda_{3}$; however, no significant correlation was obtained with any other DTI index. In the mGC at dorsal flexion, no significant correlation was obtained with any of the DTI indices. In the mGC at plantar flexion, a significant correlation was observed with $F A(r=0.478, P<0.05)$ and $\lambda_{3}(r=0.447, P<0.05)$ but not with $\lambda_{2}, \lambda_{3}$, or $A D C$. In the SOL at dorsal flexion, a significant correlation was observed with $F A(r=0.625, P<0.01)$ and $\lambda_{3}$ ( $r=0.560, P<0.05$ ) but not with $\lambda_{2}, \lambda_{3}$, or $A D C$. Likewise, in the SOL at plantar flexion, a significant correlation was observed with $F A(r=0.784, P<0.01)$ and $\lambda_{3}(r=0.600, P<0.05)$ but not with $\lambda_{2}$, $\lambda_{3}$, or $A D C$.

\section{Discussion}

Although MRI is mainly used for morphological measurements, its use for functional measurement has also attracted attention in recent years. We focused here on DTI, a technique that allows the measurement of diffusional anisotropy of water molecules. Among the skeletal muscle movements, contractions in which they actively shorten and exert force to the outside world are called concentric contractions. In the present study, we aimed to investigate the association between muscle contraction and tension resulting from this movement with measurements taken using DTI.

A lot of studies in neural area using DTI were reported, because DTI is quantitatively possible to evaluate the micro structure. By way of example, it is also used for supporting brain tumor resection surgery, which has high accuracy [23]. This structure evaluation technique has been also applied to the skeletal muscle area. It has also been reported in the studies of the assessment for muscle morphology, such as measurement of pennation angle by tensor tractography [14], depiction of orientation [15]-[17], and assessment of muscle damage [18]. Muscle structure and its function are in a tight relationship, so this study could evaluate skeletal muscle due to structural change.

1) Changes in DTI indices due to muscle contractions

The involved skeletal muscles vary among muscle movements. While the major muscles involved in plantar flexion, the gastrocnemius and SOL, were targeted in the present study, the major muscle involved in dorsal flexion is the TA. We performed DTI while these two opposing movements were performed. The fibrous cells of the skeletal muscles, called muscle fibers, measure $10-150 \mu \mathrm{m}$ in diameter and are several centimeters to several tens of centimeters in length. Muscle fibers are specialized and contain special structures for contraction. Their minimal functional unit is a contraction unit called a sarcomere, which consists of proteins called myosin and actin that slide past each other (contract) to exert tension [24]. In terms of the short axis of the myofibril, a sarcomeric contraction is an increase in the overlapping area of myosin and actin. In other words, this indicates increased myofibril density. Increased intracellular density appears to change the diffusional anisotropy of water molecules.

Our experiment showed markedly significant differences in the indices of the involved muscles between at rest and at flexion (Table 1). Significant differences were shown by the TA at dorsal flexion and by the SOL at plantar flexion. Although the mGC showed significant differences at both plantar and dorsal flexion, the differences were apparently greater at dorsal flexion (Figure 1). According to these results, DTI indices may reflect contraction and relaxation of the involved muscles (i.e. contracted muscles). In particular, $\lambda_{2}$ and $\lambda_{3}$ show greater changes. The $\lambda_{2}$ and $\lambda_{3}$ indices represent diffusion coefficients vertically to the line parallel to the skeletal muscle 
fibers $\left(\lambda_{1}\right)$ and appear to reflect increases in intracellular density due to the sliding of actin and myosin. Thus, our findings suggest that, in the assessment of muscle contraction, an analysis of indices such as $\lambda_{2}$ and $\lambda_{3}$ could more clearly reveal muscle contraction.

2) Association between muscle tension and DTI indices

In the present study, MVC was compared to DTI indices at rest. As a result, volunteers with a higher MVC showed a higher $F A$ and a lower $\lambda_{3}$. MVC was significantly correlated with $F A$ and $\lambda_{3}$ (Figure 4, Table 2). We described here that $F A$, which represents the diffusional anisotropy of water molecules in myocytes, and $\lambda_{3}$, which represents the diffusion coefficient of water molecules in the direction of the short axis, reflect increases in cell density in and out of the short axis. Accordingly, it is assumed that differences in MVC are associated with myocyte density. While it has been reported that the cross-sectional area of muscle fibers is larger in people with the ability to generate high muscle tension, PCSA has been considered an index of skeletal muscle tension [19].

PCSA is defined as the total sum of the cross-sectional areas of muscle fibers and calculated by dividing muscle volume by muscle fiber length. There is also a report that PCSA was directly calculated by measuring contiguous cross-sections obtained by MRI [25]. It was also reported that this PCSA is associated with muscle tension [26] and is proportionally associated with $\lambda_{3}$ [14]. Accordingly, PCSA appears to be associated with muscle tension. In the present study, MVC showed a significant correlation with $\lambda_{3}$, while an association between MVC and $\lambda_{3}$ was confirmed by actual measurements. Thus, it seems that intracellular and extracellular density can be assessed by FA or $\lambda_{3}$. To calculate PCSA, muscle volume and muscle fiber length should be determined. However, volume and length are extremely difficult and time-consuming to measure. The present study suggested that our proposed method to assess muscle tension using DTI enables extremely simple assessments of important factors of fiber density associated with muscle tension. Because DTI measurements take several minutes, we measured and assessed persistent muscle tension. In the future, if measurements can be performed more rapidly, instantaneous tension and kinetics such as explosive power can also be assessed.

3) Advantages and limitations

This study found a correlation between the muscle function (contraction and force) and DTI indices. Thereby, not only the structure, it was arrow for us to evaluate the motor function of muscle. Muscle function evaluation is using a dynamometer and exercise test. However, a lot of measurement biases are mixed in this test, such as conditions and environment at the time of measurement. The method in this study has a potential to evaluate the motion and the function quantitatively, which removed biases as much as possible. Skeletal muscle is a locomotorium, so the organ is changing the form at the time of the measurement state. In other words, skeletal muscle is a very important dynamic measurement. However, the method takes a few minutes for measurement so dynamic measurement is difficult. Thus, it is necessary for skeletal muscle dynamic measurement to develop the technologies such as the compressed sensing technique, which makes it possible to omit the $k$-space [27], and the hardware such as the static magnetic field and receiver coils to achieve a higher signal.

\section{Conclusion}

This study's findings suggest that skeletal muscle contractions and tension can be assessed by measuring muscle fiber contraction, muscle tension, and restricted diffusion of water molecules based on DTI indices. DTI allows not only measurements of traditionally used anatomical and morphological features but also the assessment of the functional aspects of muscles. Thus, this technique can be expected to be useful for not only disease evaluation but also the field of sports medicine.

\section{Acknowledgements}

This work was supported by Japanese Ministry of Education, Culture, Sports, Science, and Technology Grantin-Aid for Young Scientists (B) No. 26860982, 2014.

\section{References}

[1] Le Bihan, D., Mangin, J.F., Poupon, C., et al. (2001) Diffusion Tensor Imaging: Concepts and Applications. Journal of Magnetic Resonance Imaging, 13, 534-546. http://dx.doi.org/10.1002/jmri.1076

[2] Mukherjee, P., Bahn, M.M., Mckinstry, R.C., Shimony, J.S., Cull, T.S., Akbudak, E., et al. (2000) Differences between 
Gray Matter and White Matter Water Diffusion in Stroke: Diffusion-Tensor MR Imaging in 12 Patients. Radiology, 215, 211-220. http://dx.doi.org/10.1148/radiology.215.1.r00ap29211

[3] Bozzali, M., Cercignani, M., Sormani, M.P., Comi, G. and, Filippi, M. (2002) Quantification of Brain Gray Matter Damage in Different MS Phenotypes by Use of Diffusion Tensor MR Imaging. American Journal of Neuroradiology, 23, 985-988.

[4] Pfefferbaum, A., Sullivan, E.V., Hedehus, M., Lim, K.O., Adalsteinsson, E. and Moseley, M. (2000) Age-Related Decline in Brain White Matter Anisotropy Measured with Spatially Corrected Echo-Planar Diffusion Tensor Imaging. Magnetic Resonance in Medicine, 44, 259-268. http://dx.doi.org/10.1002/1522-2594(200008)44:2<259::AID-MRM13>3.0.CO;2-6

[5] Aagaard, P., Andersen, J.L., Dyhre-Poulsen, P., Leffers, A.M., Wagner, A., Magnusson, S.P., et al. (2001) A Mechanism for Increased Contractile Strength of Human Pennate Muscle in Response to Strength Training: Changes in Muscle Architecture. Journal of Physiology, 534, 613-623.

[6] Scott, S.H., Engstrom, C.M. and Loeb, G.E. (1993) Morphometry of Human Thigh Muscles. Determination of Fascicle Architecture by Magnetic Resonance Imaging. Journal of Anatomy, 182, 249-257.

[7] Bruhn, H., Frahm, J., Gyngell, M.L., Merboldt, K.D., Hanicke, W. and Sauter, R. (1991) Localized Proton NMR Spectroscopy Using Stimulated Echoes: Applications to Human Skeletal Muscle in Vivo. Magnetic Resonance in Medicine, 17, 82-94. http://dx.doi.org/10.1002/mrm.1910170113

[8] Mancini, D.M., Walter, G., Reichek, N., et al. (1992) Contribution of Skeletal Muscle Atrophy to Exercise Intolerance and Altered Muscle Metabolism in Heart Failure. Circulation, 85, 1364-1373. http://dx.doi.org/10.1161/01.CIR.85.4.1364

[9] Kemp, G.J., Taylor, D.J. and Radda, G.K. (1993) Control of Phosphocreatine Resynthesis during Recovery from Exercise in Human Skeletal Muscle. NMR in Biomedicine, 6, 66-72. http://dx.doi.org/10.1002/nbm.1940060111

[10] Takahashi, H., Kuno, S., Miyamoto, T., Yoshioka, H., Inaki, M., Akima, H., et al. (1994) Changes in Magnetic Resonance Images in Human Skeletal Muscle after Eccentric Exercise. European Journal of Applied Physiology and Occupational Physiology, 69, 408-413. http://dx.doi.org/10.1007/bf00865404

[11] Ploutz-Snyder, L.L., Nyren, S., Cooper, T.G., Potchen, E.J. and Meyer, R.A. (1997) Different Effects of Exercise and Edema on T2 Relaxation in Skeletal Muscle. Magnetic Resonance in Medicine, 37, 676-682. http://dx.doi.org/10.1002/mrm.1910370509

[12] Ploutz-Snyder, L.L., Tesch, P.A., Crittenden, D.J. and Dudley, G.A. (1995) Effect of Unweighting on Skeletal Muscle Use during Exercise. Journal of Applied Physiology, 79, 168-175.

[13] Foley, J.M., Jayaraman, R.C., Prior, B.M., Pivarnik, J.M. and Meyer, R.A. (1999) MR Measurements of Muscle Damage and Adaptation after Eccentric Exercise. Journal of Applied Physiology, 87, 2311-2318.

[14] Heemskerk, A.M., Strijkers, G.J., Vilanova, A., Drost, M.R. and Nicolay, K. (2005) Determination of Mouse Skeletal Muscle Architecture using Three-Dimensional Diffusion Tensor Imaging. Magnetic Resonance in Medicine, 53, 13331340. http://dx.doi.org/10.1002/mrm.20476

[15] Budzik, J.F., Le Thuc, V., Demondion, X., Morel, M., Chechin, D. and Cotton, A. (2007) In Vivo MR Tractography of Thigh Muscles Using Diffusion Imaging: Initial Results. European Radiology, 17, 3079-3085. http://dx.doi.org/10.1007/s00330-007-0713-z

[16] Kan, J.H., Heemskerk, A.M., Ding, Z., Gregory, A., Mencio, G., Spindler, K. and Damon, B.M. (2009) DTI-Based Muscle Fiber Tracking of the Quadriceps Mechanism in Lateral Patellar Dislocation. Journal of Magnetic Resonance Imaging, 29, 663-670. http://dx.doi.org/10.1002/jmri.21687

[17] Sinha, S., Sinha, U. and Edgerton, V.R. (2006) In Vivo Diffusion Tensor Imaging of the Human Calf Muscle. Journal of Magnetic Resonance Imaging, 24, 182-190. http://dx.doi.org/10.1002/jmri.20593

[18] McMillan, A.B., Shi, D., Pratt, S.J. and Lovering, R.M. (2011) Diffusion Tensor MRI to Assess Damage in Healthy and Dystrophic Skeletal Muscle after Lengthening Contractions. Journal of Biomedicine and Biotechnology, 2011, Article ID: 970726. http://dx.doi.org/10.1155/2011/970726

[19] Wickiewicz, T.L., Roy, R.R., Powell, P.L., Perrine, J.J. and Edgerton, V.R. (1984) Muscle Architecture and ForceVelocity Relationships in Humans. Journal of Applied Physiology, 57, 435-443.

[20] Lehnert, A., Machann, J., Helms, G., Claussen, C.D. and Schick, F. (2004) Diffusion Characteristics of Large Molecules Assessed by Proton MRS on a Whole-Body MR System. Magnetic Resonance Imaging, 22, 39-46. http://dx.doi.org/10.1016/j.mri.2003.05.007

[21] Hata, J., Yagi, K., Hikishima, K, Numano, T., Goto, M. and Yano, K. (2013) Characteristics of Diffusion-Weighted Stimulated Echo Pulse Sequence in Human Skeletal Muscle. Radiological Physics and Technology, 6, 92-97.

http://dx.doi.org/10.1007/s12194-012-0174-1 
[22] Basser, P. and Pierpaoli, C. (1996) Microstructural and Physiological Features of Tissues Elucidated by QuantitativeDiffusion-Tensor MRI. Journal of Magnetic Resonance, 111, 209-219. http://dx.doi.org/10.1006/jmrb.1996.0086

[23] Suzuki, Y., Yagi, K., Kodama, T. and Shinoura, N. (2009) Corticospinal Tract Extraction Combining Difusion Tensor Tractography with fMRI in Patients with Brain Diseases. Magnetic Resonance in Medicine, 8, 9-16. http://dx.doi.org/10.2463/mrms.8.9

[24] Gordon, A.M., Huxley, A.F. and Julian, F.J. (1966) The Variation in Isometric Tension with Sarcomere Length in Vertebrate Muscle Fibres. Journal of Physiology, 184, 170-192. http://dx.doi.org/10.1113/jphysiol.1966.sp007909

[25] Fukunaga, T., Roy, R.R., Shellock, F.G., Hodgson, J.A., Day, M.K., Lee, P.L., Kwong-Fu, H. and Edgerton, V.R. (1992) Physiological Cross-Sectional Area of Human Leg Muscles Based on Magnetic Resonance Imaging. Journal of Orthopaedic Research, 10, 928-934. http://dx.doi.org/10.1002/jor.1100100623

[26] Fukunaga, T., Ichinose, Y., Ito, M., Kawakami, Y., Fukashiro, S., et al. (1997) Determination of Fascicle Length and Pennation in a Contracting Human Muscle in Vivo. Journal of Applied Physiology, 82, 354-358.

[27] Lustig, M., Donoho, D. and Pauly, J.M. (2007) Sparse MRI: The Application of Compressed Sensing for Rapid MR Imaging. Magnetic Resonance in Medicine, 58, 1182-1195. http://dx.doi.org/10.1002/mrm.21391 